\title{
Local-Field Distribution in Random Dielectric Media
}

\author{
Ping Sheng and Zhe Chen ${ }^{(a)}$ \\ Exxon Research and Engineering Company, Annandale, New Jersey 08801 \\ (Received 9 July 1987)
}

\begin{abstract}
By generalizing Onsager's reaction-field approach, we calculate the local field in random dielectric media characterized by uniform or percolative correlations. The distribution of the local field is found to display a double-peak character in general. We show that the variance of the distribution is linearly correlated to the deviation of the mean from the Lorentz value, where the latter quantity is also identified as the correlation to the Clausius-Mossotti relation. Structural correlations are demonstrated to have a significant effect in determination of the distribution characteristics.
\end{abstract}

PACS numbers: 78.20.Dj, 41.10.- j, 42.20.-y, 77.20.+y

The Lorentz local field ${ }^{1}$ and the Clausius-Mossotti (CM) relation have long been recognized as the foundation of our study of dielectrics as well as the basis of generalization for effective-medium theories ${ }^{2-4}$ that were widely applied to inhomogeneous composites. In recent years, experiments involving the physical phenomena such as surface-enhanced Raman scattering, ${ }^{5}$ fluorescence, ${ }^{6}$ and surface reflectance anisotropy ${ }^{7-9}$ have called attention to the particularly important role of microstructural inhomogeneities in determining the local field and its spatial variations. While the idea of spatialfluctuation correction to the Lorentz local field and the CM relation is not new and has been explored theoretically $^{10-13}$ in general terms, yet in view of the recent advances in the understanding of the structure and symmetry of random physical systems, ${ }^{14-16}$ there has still been no systematic investigation of their local-field and dielectric consequences. In particular, the local-field implications of extended structural correlations, which is a common feature for many random systems, remain to be addressed.

In this Letter, we study the local field and effective dielectric properties of a lattice gas of polarizable point particles whose structure is characterized by either uniform or percolative correlations. Physically, the uniform correlation case can correspond to a gas or a fluid or solid mixture of two atomic species. The percolative structure, on the other hand, can correspond to materials that result from growth or aggregation processes. By generalizing Onsager's reaction-field approach, ${ }^{17}$ we obtain for the first time the distribution of local field and its variation with density and geometry. We find the distri- bution to be doubly peaked in general, with the Lorentz value, $E_{\mathrm{L}}$, situated between the two peaks. Furthermore, the variance of the distribution is shown to be linearly correlated with the shift of the mean from the Lorentz value. The shift, in turn, is also identified as the correction to the CM relation. In terms of these distribution characteristics, the percolative structure is found to yield significantly different behavior from those with the uniform correlation.

Consider an inhomogeneous system consisting of polarizable point particles randomly occupying the sites of a simple cubic lattice. In general, a random medium is characterized by a correlation length $\zeta$, a scale on (or beyond) which the medium may be regarded as homogeneous. To calculate the local-field distribution that is representative of the inhomogeneous system, we would like to solve the electrostatic problem of $N$ particles inside a spherical cavity of radius $a>\zeta$ under the action of an applied field. The material outside the cavity is assumed to be homogeneous and characterized by an as yet unspecified dielectric constant $\epsilon$. Following Onsager, ${ }^{17}$ we express the local field $\mathbf{E}$ acting on the $i$ th particle as

$$
E_{\mu}\left(\mathbf{r}_{i}\right)=G_{\mu}\left(\mathbf{r}_{i}\right)+\sum_{j}^{N} R_{\mu}\left(\mathbf{r}_{i}, \mathbf{r}_{j}\right)+\sum_{j \neq i}^{N} D_{\mu}\left(\mathbf{r}_{i}, \mathbf{r}_{j}\right),
$$

where $G_{\mu}, R_{\mu}$, and $D_{\mu}$ stand for the components of the cavity field, the reaction field, and the dipolar field, respectively; and $\mathbf{r}_{i}$ denotes the position of particle $i$ inside the cavity. The form of $R_{\mu}$ may be obtained by the solution of the electrostatic problem of a dipole located at arbitrary position inside the cavity. By our further noting that $\mathbf{G}=[3 \epsilon /(2 \epsilon+1)] \mathbf{E}^{0}$, where $\mathbf{E}^{0}$ is the macroscopic field, and $\mathbf{r}_{i j}=\mathbf{r}_{i}-\mathbf{r}_{j}$, we get

$$
\begin{aligned}
& \sum_{v=1}^{3} \sum_{j=1}^{N} S_{\mu v}\left(\mathbf{r}_{i}, \mathbf{r}_{j}\right) E_{v}\left(\mathbf{r}_{j}\right)+[3 \epsilon /(2 \epsilon+1)] E_{\mu}^{0}\left(\mathbf{r}_{i}\right)=E_{\mu}\left(\mathbf{r}_{i}\right), \\
& S_{\mu v}\left(\mathbf{r}_{i}, \mathbf{r}_{j}\right)=\sigma_{\mu v}\left(\mathbf{r}_{i}, \mathbf{r}_{j}\right)+\gamma_{\mu v}\left(\mathbf{r}_{i}, \mathbf{r}_{j}\right)\left(1-\delta_{i j}\right) \\
& \sigma_{\mu v}\left(\mathbf{r}_{i}, \mathbf{r}_{j}\right)=\alpha \sum_{l=0 m}^{\infty} \sum_{l}^{l} c_{l m} \partial_{\mu} q\left(l, m, \mathbf{r}_{i}\right) \partial_{v} q^{*}\left(l, m, \mathbf{r}_{j}\right)
\end{aligned}
$$


where

$$
q\left(l, m, \mathbf{r}_{i}\right)=a^{-l}\left|r_{i}\right|^{l} Y_{l m}\left(\Theta_{i}, \phi_{i}\right)
$$

$Y_{l m}$ are the spherical harmonics,

$$
c_{l m}=\frac{(\epsilon-1)(l+1)}{l+(l+1) \epsilon} a^{-1}\left(\frac{4 \pi}{2 l+1}\right)^{3 / 2}\left[\frac{(l+m) !}{(l-m) !}\right]^{1 / 2}
$$

and $\gamma_{\mu \nu}=\alpha \partial_{\mu} \partial_{\nu} r_{i j}^{-1}$. Equations (2) constitute a set of $3 N$ linear simultaneous equations with the polarizability $\alpha$, the coordinates of the particles, and $\epsilon$ as inputs. To obtain $\epsilon$, we regard the system as composed of units each of which has a size $\sim 2 a$. The dielectric constant may then be determined by the coherent-potential approximation $^{3,4}$ (or the effective-medium theory) which, in the static limit, is simply the condition that the average dipole moments of all the units should vanish. Straightforward algebra yields

$$
\frac{\epsilon-1}{4 \pi}=\rho \alpha\left\langle\frac{1}{N} \sum_{i=1}^{N} \mathrm{E}\left(\mathbf{r}_{i}\right) \cdot \hat{\mathbf{e}} / E^{0}\right\rangle=\rho \alpha \mathrm{E}_{m} \cdot \hat{\mathbf{e}} / E^{0},
$$

where $\rho$ is the site occupation fraction, $\hat{\mathbf{e}}$ the unit vector along the applied field $\mathbf{E}^{0}, \mathbf{E}_{m}$ is the mean of the local field, and angular brackets denote configurational averaging. Equations (2) and (3) form the self-consistent basis of the generalized Onsager approach to the calculation of local fields and dielectric constant. It is easy to verify that if the spherical cavity contains only one particle, i.e., $a \simeq$ lattice constant, then one recovers the Lorentz formula and the CM relation.

For calculation on random systems, a cavity of radius $a>\zeta$ is drawn with the positions of particles inside as inputs to Eq. (2). The cavity radius $a$ is slightly adjusted so as to make the particle density inside the cavity correspond as closely as possible with the mean density. For those particles lying close to the boundary of the cavity, the interface can produce an artificial distortion of the local environment. To mitigate this effect, we use the rule that the local field of any particle $j$ which is closer than $\alpha^{1 / 3}$ from the boundary is assigned a value of $\mathbf{E}_{m}$, i.e., $\mathbf{E}\left(\mathbf{r}_{j}\right)=\mathbf{E}_{m}$ in Eqs. (2). Equations (2) and (3) are then solved self-consistently by iteration. To calibrate our method, we first examine the case of $\rho=1$ where it is known that the Lorentz formula is exact. Indeed, local fields for $N$ particles $(N>27)$ are all identical and equal to the Lorentz value $E_{\mathrm{L}}=(\epsilon+2) E^{0 / 3}$ no matter where one chooses the center of the cavity. For homogeneous random occupation of the sites, i.e., uniform correlation, the same $\delta$-function-like distribution is also found for $\rho \simeq 0$. At intermediate values of $\rho$, three representative distributions ${ }^{18}$ calculated with $\alpha=0.1$ (in units of lattice constant cubed) are displayed in Figs. $1(\mathrm{a})-1$ (c) as a function of $E_{\|} / E_{\mathrm{L}}$, where $E_{\|}=\mathbf{E} \cdot \hat{\mathbf{e}}$. It is seen that the distribution $P_{\rho}\left(E_{\|} / E_{\mathrm{L}}\right)$ generally exhibits two peaks, ${ }^{19}$ with the single peak in the $\rho=0.5$ case also interpretable

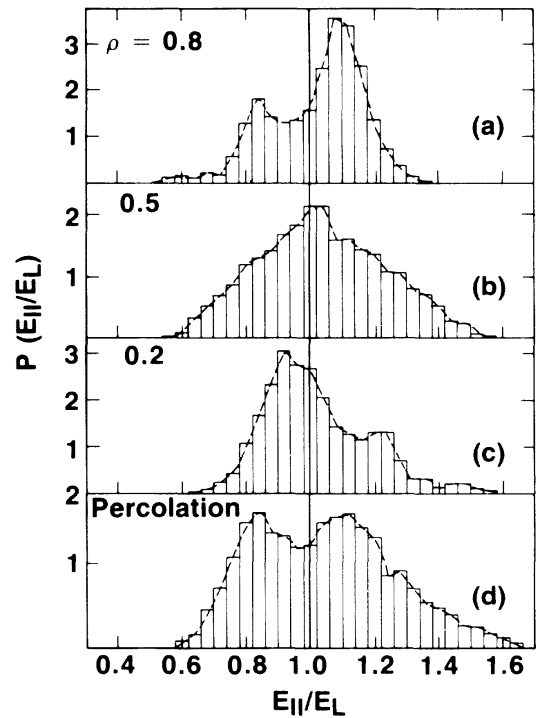

FIG. 1. Local-field distribution plotted as a function of $E_{\|} / E_{\mathrm{L}}$. (a)-(c) are for uniform but random systems of different densities. (d) is for a random percolative structure. Number of particles in each of the distributions is (a) 2593, (b) 3117 , (c) 3103 , and (d) 5613.

as the merging of the two. It can be easily verified that the peak above $E_{\mathrm{L}}$ arises from a local environment where the dipole field contributions from the neighbors above and below the site (in the direction of the field) exceed those in the plane of the site, which we denote as a type1 environment, whereas the peak below $E_{\mathrm{L}}$ arises from the reverse situation, which we denote as a type- 2 environment. In Fig. 1(d) we show the result for a percolative structure (obtained by retaining only "connected" sites) with an occupation fraction of $\rho=0.261, \alpha=0.1$, and a correlation length $\zeta \simeq 6.4$ (lattice units). The double-peak character of the distribution is even more pronounced with an accompanying increase in the distribution width. It should be remarked that since the system is linear and macroscopically equivalent in the three coordinate-axis directions, the phenomena discussed above are invariant with respect to the orientation of the applied field. Also, to check the effect of the underlying lattice, we have calculated local fields for clusters that are generated on fcc and hcp lattices. Qualitatively similar results were obtained.

Since the deviations from the Lorentz local-field value can be attributed to the field of dipoles inside the Lorentz cavity, ${ }^{1}$ a first-order estimate for the magnitude of the deviation may be obtained from the parameter

$$
\eta=\left\langle\left|\sum_{j \neq i} \mathbf{D}\left(\mathbf{r}_{i}, \mathbf{r}_{j}\right)\right|^{2}\right\rangle^{1 / 2},
$$

where the dipole field $\mathbf{D}$ at site $i$ is calculated by our assuming that the dipole at every site is given by $\mathbf{p}=\hat{\mathbf{e}}$, and the summation is over all the occupied sites $j$ inside a suitably large sphere (so that the value of $\eta$ converges) 


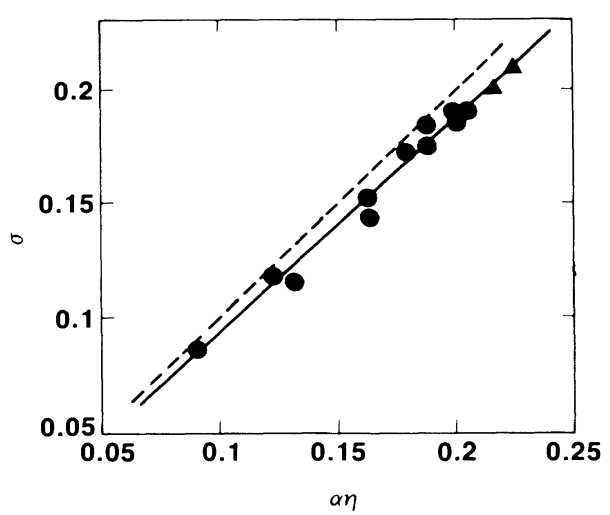

FIG. 2. Standard deviation of the local-field distribution plotted as a function of $\alpha \eta$, where $\eta$ is defined in the text. Circles are for uniform correlations with each point representing the average of 1500-3000 particles; triangles denote the results for percolative structures where each point represents the average over $3000-6000$ particles. It is seen that the percolative structures yield larger variances. The solid line is the leastsquares fit given by Eq. (5). The dashed line, representing $\sigma=\alpha \eta$, is drawn for reference.

centered at $i$. While the assumption that the induced dipole is the same at every occupied site is inconsistent with the actual situation, one can nevertheless attribute a physical meaning to the parameter $\eta$. From symmetry, we know that $\eta=0$ for a cubic or spherical environment as assumed in the Lorentz formula, and $\eta \neq 0$ in general for random systems. Therefore, $\eta$ may be regarded as a geometric parameter that measures the local anisotropy in the dipolar sense. In Fig. 2 the standard deviation $\sigma=\left\langle\left(E_{\|} / E_{m}\right)^{2}-1\right\rangle^{1 / 2}$ is plotted versus the computed $\alpha \eta$ value for every calculated sample (including those not shown in Fig. 1). A least-squares fit by the equation $\sigma=a+b \alpha \eta$ yields

$$
\sigma=0.94 \alpha \eta
$$

with $a \sim 10^{-4}$. The accuracy of the linear relation tells us that the standard deviation of the distribution is directly correlated with the degree of local anisotropy.

From Eq. (3) it is seen that the mean of the local field, $\mathbf{E}_{m}$, is directly related to the dielectric constant of the system. We can reexpress Eq. (3) in a slightly different form:

$$
(\epsilon-1) /(\epsilon+2)=\frac{4}{3} \pi \rho \alpha(1+S),
$$

which is precisely the $C M$ relation with a correction term $^{20,21} S=\Delta E / E_{\mathrm{L}}$, where $\Delta E=\mathrm{E}_{m} \cdot \hat{\mathbf{e}}-E_{\mathrm{L}}$. In Fig. 3 we plot in filled symbols the calculated corrections $S / \beta^{2}$ as a function of $\rho$ for both uniform and percolative random structures. The constant $\beta^{2}$ is defined as ${ }^{20} B \alpha^{2}$, where the constant $B$ is $11.202 \pi$ for our model and $16 \pi^{2} / 9$ for a system of hard spheres. Division of $S$ by $\beta^{2}$ removes the leading-order dependence on $\alpha$ and the underlying lattice and makes possible direct comparison

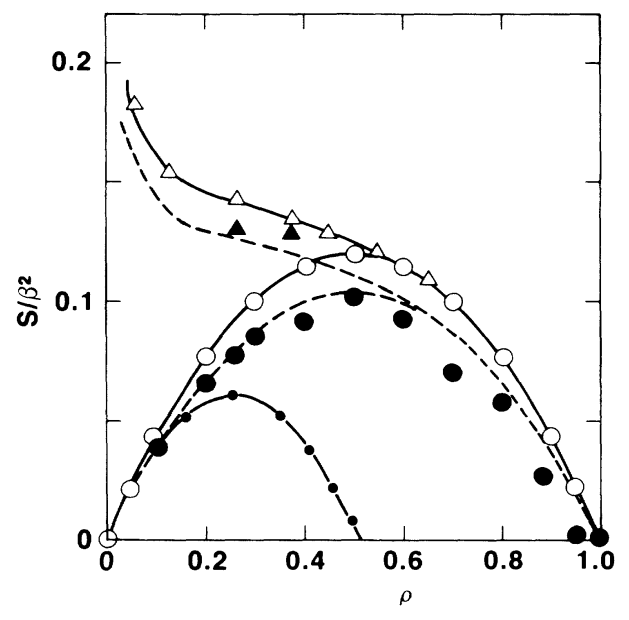

FIG. 3. Correction to the Clausius-Mossotti relation plotted as a function of density. Filled symbols represent the results obtained with use of the generalized Onsager approach with the same statistics as that for Fig. 2. Open symbols denote $S^{(2)}$ calculated from the theory of Kirkwood where each point is the average of 30000-100000 particles. Circles are for uniform systems; triangles are for percolative structures. In descending order, the correlation length for the calculated percolative structures are (in terms of lattice constant) 176, 23, $6.4,3.4,2.3,1.5$, and 1.1. The dash-dotted line is obtained from Kirkwood's low-density expansion (Ref. 10). The dashed lines are calculated from the relation $S=\sigma^{2}$.

with literature results. The first characteristic one notices about $S$ is that it is nonnegative, i.e., $\Delta E \geq 0$. This could be understood by consideration of the theory of Kirkwood, ${ }^{10}$ in which one can show that the leadingorder term for $S, S^{(2)}$, is expressible as $\alpha^{2} \eta^{2}$. It follows that if the higher-order corrections are small, then $S \geq 0$. The computed $S^{(2)}$ is plotted in Fig. 3 as open symbols connected by solid lines. ${ }^{22}$ The good agreement with the calculated values of $S$ demonstrates that $S^{(2)}$ is the major fraction of $S$. Kirkwood's analytic expan$\operatorname{sion}^{10}$ of $S^{(2)}$ for a system of low-density hard spheres is also plotted for comparison. A curious implication of the fact $\mathbf{E}_{m} \cdot \hat{\mathbf{e}} \geq E_{\mathrm{L}}$ is that if an initially simple cubic material is randomized, then the net absorption of the material would increase because the mean of the local fields does not average to the same value as before but would always increase. Furthermore, since $S^{(2)}$ is proportional to $\eta^{2}$, the amount of increase is governed by the degree of local anisotropy. Another interesting suggestion from the fact that $S=S^{(2)}=\alpha^{2} \eta^{2}$ is that the deviation from the CM relation offers, via Eq. (5), a direct measure of the variance for the local-field distribution. To test the general validity of this proposition, we plot in Fig. 3 the dashed lines calculated from the relation $S=\sigma^{2}$ $=0.883 \alpha^{2} \eta^{2}$. Excellent agreement is obtained with the calculated solid points to within statistical errors. The existence of the correlation $S=\sigma^{2}$ implies that if the mean of the local field is given by the Lorentz value, then 
the distribution must be a $\delta$ function. As the mean increases away from the Lorentz value, the distribution also broadens proportionally.

The difference between the uniform and the percolative structures is clearly visible in Fig. 3: Whereas the uniform random system has the maximum CM correction at $\rho=0.5$, the percolative structure with extended structural correlations shows monotonic increases in the value of $S$ as the correlation length increases. In fact, a finite limiting value of $S$ is predicted for $\rho=0$ (and $\zeta \rightarrow \infty)$ which would be that of the percolation cluster. Since the increase in $S$ at low densities arises from increased local anisotropies, we expect the local-field distributions for aggregate or growth structures to have large variances. The Lorentz local-field value would therefore be a very poor approximation in these systems. For a polarizable fractal structure with fractal dimension $<3, \zeta \rightarrow \infty$ (and therefore $\rho \rightarrow 0$ ), we have the interesting case of $\epsilon \rightarrow 1$ and yet the local field is predicted to deviate significantly from $E_{\mathrm{L}}=E^{0}$. Implications of our results for the effective-medium theories are presently being pursued.

\footnotetext{
(a) Also at Department of Physics, City College of New York, New York, NY 10031.

${ }^{1}$ See, for example, C. Kittel, Introduction to Solid State Physics (Wiley, New York, 1976), 5th ed., p. 399.

${ }^{2} \mathrm{R}$. Landauer, in Electrical Transport and Optical Properties of Inhomogeneous Media-1977, edited by J. C. Garland and D. B. Tanner, AIP Conference Proceedings No. 40 (American Institute of Physics, New York, 1978), p. 2.

${ }^{3}$ D. Stroud and F. P. Pan, Phys. Rev. B 17, 1602 (1978).

${ }^{4} \mathrm{P}$. Sheng, in Homogenization and Effective Moduli of Ma-
}

terials and Media, edited by J. L. Ericksen et al., IMA Volumes in Mathematics and Its Applications Vol. 1 (Springer-Verlag, New York, 1986), p. 196.

${ }^{5}$ For a review, see S. G. Schultz, M. Janik-Czachor, and R. P. Van Duyne, Surf. Sci. 104, 419 (1981).

${ }^{6}$ H. Chew, D. D. Cooke, and M. Kerker, Appl. Opt. 19, 44 (1980).

${ }^{7}$ D. E. Aspnes and A. A. Studna, Phys. Rev. Lett. 54, 1956 (1985).

${ }^{8}$ W. L. Mochán and R. G. Barrera, Phys. Rev. Lett. 56, 2221 (1986).

${ }^{9}$ W. L. Mochán and R. G. Barrera, Phys. Rev. Lett. 55, 1192 (1985).

10 J. G. Kirkwood, J. Chem. Phys. 4, 592 (1936).

${ }^{11}$ D. E. Aspnes, Phys. Rev. Lett. 48, 1629 (1982).

${ }^{12}$ G. W. Milton, Phys. Rev. Lett. 46, 542 (1981).

${ }^{13}$ W. L. Mochán and R. G. Barrera, Phys. Rev. B 32, 4984 (1985)

${ }^{14}$ T. A. Witten, Jr., and L. M. Sander, Phys. Rev. Lett. 47, 1400 (1981).

${ }^{15}$ D. A. Weitz and M. Oliveria, Phys. Rev. Lett. 52, 1433 (1984).

${ }^{16}$ R. Zallen, The Physics of Amorphous Solids (Wiley, New York, 1983).

${ }^{17}$ L. Onsager, J. Am. Chem. Soc. 58, 1486 (1936).

${ }^{18}$ The distribution for $E_{\perp}=|\mathbf{E} \times \hat{\mathbf{e}}|$ is a half Gaussian with peak at $E_{\perp}=0$ and a half width on the order of $0.01 E^{0}$.

${ }^{19}$ Since the local field is calculated on occupied sites only and the polarizable particle has no internal states such as that for the Ising spin the double-peak character here should be distinguished from those model behaviors which originate from different types of sites or internal states.

${ }^{20}$ J. DeBoer, F. Van der Maesen, and C. A. Ten Seldam, Physica (Utrecht) 19, 2655 (1953).

${ }^{21}$ G. Stell and G. S. Rushbrooke, Chem. Phys. Lett. 24, 531 (1974).

${ }^{22}$ To a high degree of accuracy, we have $S^{(2)} / \beta^{2}$ $=0.48 \rho(1-\rho)$ for the uniform random case. 